\title{
ERRATUM
}

Open Access

\section{Erratum to: Scale development: ten main limitations and recommendations to improve future research practices}

Fabiane F. R. Morgado ${ }^{1 *}$, Juliana F. F. Meireles ${ }^{2}$, Clara M. Neves ${ }^{2}$, Ana C. S. Amaral ${ }^{3}$ and Maria E. C. Ferreira ${ }^{2}$

\section{Erratum}

The original publication [1] contains an error in Table 1.

The correct version:

Medina-Pradas et al. (2011): "EFA/CV/CtV/ICR"

\author{
Author details \\ 'Institute of Education, Universidade Federal Rural do Rio de Janeiro, BR-465, \\ km 7, Seropédica, Rio de Janeiro 23890-000, Brazil. Faculty of Psychology, \\ Universidade Federal de Juiz de Fora, Rua José Lourenço Kelmer, s/ n-Campus \\ Universitário Bairro São Pedro, Juiz de Fora, Minas Gerais 36036-900, Brazil. \\ ${ }^{3}$ Faculty of Physical Education of the Instituto Federal de Educação, Ciência e \\ Tecnologia do Sudeste de Minas Gerais, Av. Luz Interior, n 360, Estrela Sul, Juiz \\ de Fora, Minas Gerais 36030-776, Brazil.
}

Received: 17 February 2017 Accepted: 17 February 2017

Published online: 03 March 2017

\section{Reference}

1. Scale development: ten main limitations and recommendations to improve future research practices Psicologia: Reflexão e Crítica Psychology: Research and Review 2017 30:3 DOI: 10.1186/s41155-016-0057-1.

\footnotetext{
* Correspondence: fabi.frm@hotmail.com

${ }^{1}$ Institute of Education, Universidade Federal Rural do Rio de Janeiro, BR-465, km 7, Seropédica, Rio de Janeiro 23890-000, Brazil
} 